\title{
Evaluation of Genetic Variability of Faba Bean (Vicia faba L.) Genotypes under Different Environments
}

\author{
Rehab A. M. Abdel-Rahman', A. A. Abou-Zeid ${ }^{1}$ and Wafaa W. M. Shafei ${ }^{2}$ \\ ${ }^{1}$ Food Legume Crops Res. Dep., Field Crops Res. Inst., ARC, Giza. \\ ${ }^{2}$ Central Laboratory for Design \& Statistical Analysis Research, ARC, Giza.
}

\begin{abstract}
The development of high yielding cultivars is a primary objective in most faba bean breeding program. This study is an attempte to evaluate sixty faba bean genotypes under different environment; Sids Research Station in Middle Egypt and Nubaria Research Station in North Egypt, during winter growing seasons 2014/2015 and 2015/2016.The experimental design was an alpha lattice with two replications. The combined analysis of variance over years were significant for most trait. The results for plant height showed that G2 and G50 had the tallest genotypes at Nubaria and Sids locations, respectively. The G42 recorded the highest values for number of pod, seeds and seed weight.plant ${ }^{-1}$ at Sids location, whereas, G18 genotype recoded the highest number of pods. Plant ${ }^{-1}$ at Nubaria location. G49 and G40 recorded the heaviest 100 seed weight at Sids location, while the genotype G51 had the heaviest value at Nubaria location. Regarding seed yield $\left(\operatorname{ardab} . \mathrm{Fad}^{-1}\right)$, the genotypes G27and G45 gave the highest values at Sids and Nubaria locations, respectively. The cluster analysis classified the tested genotypes in both locations to four main different groups, the first group had the highest seed yielding $\left(\operatorname{ardab} . \mathrm{fad}^{-1}\right)$, plant height and seed weight. Plant ${ }^{-1}$, followed by the third group cluster in the location Sids.
\end{abstract}

Key wards: alpha lattice, cluster analysis, genotypes, faba bean.

\section{INTRODUCTION}

Faba bean (Vicia faba, L.)is one of the most important food and feed legumes in the world. In Mediterranean countries and China, Faba bean provides a valuable source of cheep protein for food. In developed countries, it is mainly used as a feed. Also, Faba bean contributes to farmers income and improves soil fertility through biological nitrogen fixation.

A rich and diverse germplasm collection is the backbone of successful crop improvement program. Genetic resources have played a major role in providing source of resistance to biotic and a biotic stresses. It is important not only to collect genetic resources, but also to evaluate, document and utilize for immediate and long-term breeding programs.

The world cultivated area by faba bean was 2.55 million hectares, which yielded a total output of 4.3 million tons (FAO, 2012). In Egypt, the average of cultivated area over the last caple of years (2012-2018) was about 113.000 Faddan with an average yield of 9.2 ardab. Fad ${ }^{-1}$ with a total production of 119,000 ton, whereas, the consumption reached 420,000 ton. It is required to expand faba beans production to meet the high demotic demands. This expansion, forced to takeplace in newly reclaimed areas (Bakry et al, 2011).

Suitable experimental design is a basic tool for validating error term of differences. Multienvironments experiments require wise choice of design and data manuplation.

The goal of this study was to evaluate newly developed promising faba bean genotypes under variable environmental conditions using alpha lattice experimental design and calculate similarity and dissimilarity parameters among genotypes using cluster analysis

\section{MATERIALS AND METHODS}

Two field experiments were carried out at two different locations; Sids Research Station in Middel Egypt and Nubaria Research Station in North Egypt, ARC, during the two growing seasons 2014/2015 and 2015/2016, to evaluate sixty faba bean genotypes . Soil analysis of experimental sites were presented in Table (1). The sixty tested genotypes (from G1 to G60) were four Egyptian commercial cultivars (Giza 429, Misr 3, Sakha 1 and Giza 834), and 56 genotypes developed by the Legume Crops Research Section, Agricultural Research Center, Egypt. The name and pedigree of faba bean genotypes were presented in Table 2 .

Faba bean genotypes were laid out in a combined an alpha lattice design with two replications as described by Patterson and Williams (1976). Each replication was divided into five incomplete blocks with twelve plots for each. The layout of the field experiment was a grid of 10 rows and 12 columns in the two growing seasons and two locations as shown in Table. (3). The randomization of 60 genotypes was done with GenStat v.14 software (Payne et al., 2011).

Each plot consisted of four ridges, three $\mathrm{m}$. long and $0.6 \mathrm{~m}$ apart (plot area $=7.2 \mathrm{~m}^{2}$ ) with one seed hill-1,20 cm apart. Seeds of all genotypes were inoculated and hand planted at density of 15 plants per ridge. All other agricultural practices were maintained at optimum levels to maximize faba bean productivity. At maturity, ten guarded plants 
were taken at random from each experimental plot for each genotype. The following data were recorded: plant height $(\mathrm{cm})$, number of branches, pods, seeds and seed weight. Plants 100 -seed weight (g) and seed yield $\left(\operatorname{ardab} \mathrm{fad}^{-1}\right)($ oneardab $=155 \mathrm{~kg}$ and one faddan $=4200 \mathrm{~m}^{2}$ ).

\section{Statistical analysis Methods}

Combined Alpha lattice designs are partially balanced designs because some pairs of genotypes did not meet in any incomplete block (termed as 0 ), other pairs of genotypes came together in the incomplete blocks once (termed as 1), others came together twice (termed as 2). Accordingly, there are many available orders of alpha lattice designs but the two orders of $(0,1)$ and $(0,1,2)$ are considered the most efficient and accurate structures. In the current work, the layout plan of the 60 tested genotypes followed the order of $(0,1)$. Data of seed yield was separately analysized for each year as well as combined analysis over the two years in each location (Patterson and Williams, 1976) after insurance of homogeneity of individual error term. However, the valid standard error was used to estimate the least significant difference (LSD) to compare each pair-wise genotype means.

Cluster analysis: Cluster analysis is driven by the trade of between minimizing the Euclidean distance of observation within a cluster and maximizing the Euclidean distance between clusters (Vural and Karasu, 2007). This was done by SPSS software (Ver. 16.0.1, SPSS Inc), to find groups of genotypes on the basis of coordinates of principal component analysis.

Table 1: Soil physic-chemical properties of the experimental two sites (Water Soils, and Environment Research Institute, ARC, Giza).

\begin{tabular}{|c|c|c|}
\hline \multirow{2}{*}{ Soil properties } & \multicolumn{2}{|c|}{ Location } \\
\hline & Sids & Nubaria \\
\hline \multicolumn{3}{|l|}{ Mechanical analysis : } \\
\hline Sand \% & 9.5 & 52.41 \\
\hline Silt \% & 31.9 & 27.64 \\
\hline Clay \% & 60.6 & 19.67 \\
\hline Texture grand & Clay & Sandy loam \\
\hline \multicolumn{3}{|l|}{$\underline{\text { Soil analysis }}$} \\
\hline S.P. \% & 48.77 & 31.6 \\
\hline $\mathrm{P}^{\mathrm{H}}$ & 7.72 & 8.24 \\
\hline E.C. dSm & 1.04 & 1.73 \\
\hline Organic Carbon \% & 0.53 & 0.13 \\
\hline Organic Matter \% & 0.91 & 0.21 \\
\hline $\mathrm{CaCo}_{3} \%$ & 11.61 & 22.63 \\
\hline Soluble Nitrogen(mg.kg) & 62.46 & 14.72 \\
\hline Total Nitrogen \% & 0.028 & 0.014 \\
\hline Available_P \% & 7.62 & 3.23 \\
\hline Available_K \% & 311.60 & 107.9 \\
\hline \multicolumn{3}{|l|}{ EDTA_extractable: } \\
\hline $\mathrm{Fe} \quad \mathrm{ppm}$ & 8.60 & 3.2 \\
\hline Mn $\quad$ ppm & 4.31 & 0.19 \\
\hline $\mathrm{Zn} \quad \mathrm{ppm}$ & 4.10 & 0.21 \\
\hline $\mathrm{Cu} \quad \mathrm{ppm}$ & 1.81 & 0.17 \\
\hline \multicolumn{3}{|l|}{ Soluble Cations $\left(\mathrm{meql}^{-1}\right)$ : } \\
\hline $\mathrm{Ca}^{++}$ & 3.00 & 4.18 \\
\hline $\mathrm{Mg}^{++}$ & 1.36 & 1.62 \\
\hline $\mathrm{Na}^{+}$ & 5.12 & 9.36 \\
\hline $\mathrm{K}^{+}$ & 0.98 & 2.14 \\
\hline \multicolumn{3}{|l|}{ Soluble Anions $\left(\mathrm{meql}^{-1}\right)$ : } \\
\hline $\mathrm{CO}_{3}^{--}$ & 0.00 & 0.00 \\
\hline $\mathrm{HCO}_{3}^{-}$ & 1.51 & 3.95 \\
\hline $\mathrm{Cl}^{-}$ & 1.72 & 11.7 \\
\hline $\mathrm{SO}_{4}^{--}$ & 7.23 & 1.74 \\
\hline
\end{tabular}


Table 2: The name and pedigree of the sixy faba bean genotypes.

\begin{tabular}{|c|c|c|c|c|c|}
\hline Code & Genotypes & Pedigree & Code & Genotypes & Pedigree \\
\hline G1 & 2097/391/2014 & Nubaria 2 x Cairo 5 & G31 & $2114 / 471 / 2014$ & Misr 3 x Cross 1906 \\
\hline G 2 & $2097 / 392 / 2014$ & Nubaria $2 \times$ Cairo 5 & G32 & $2114 / 472 / 2014$ & Misr 3 x Cross 1906 \\
\hline G 3 & $2104 / 395 / 2014$ & Nubaria 3 x Cairo 4 & G33 & $2114 / 473 / 2014$ & Misr 3 x Cross 1906 \\
\hline G 4 & $2104 / 399 / 2014$ & Nubaria 3 x Cairo 4 & G34 & $2115 / 475 / 2014$ & Misr 3 x Cross 1907 \\
\hline G 5 & $2104 / 400 / 2014$ & Nubaria 3 x Cairo 4 & G35 & $2115 / 476 / 2014$ & Misr 3 x Cross 1907 \\
\hline G 6 & $2105 / 407 / 2014$ & Nubaria $3 \times$ Cairo 25 & G36 & $2115 / 477 / 2014$ & Misr 3 x Cross 1907 \\
\hline G7 & $2106 / 410 / 2014$ & Nubaria 3 x Cross 1714 & G37 & $2115 / 478 / 2014$ & Misr 3 x Cross 1907 \\
\hline G8 & $2107 / 413 / 2014$ & Nubaria 3 x Cross 1906 & G38 & $2115 / 479 / 2014$ & Misr 3 x Cross 1907 \\
\hline G9 & $2107 / 414 / 2014$ & Nubaria 3 x Cross 1906 & G39 & $2115 / 480 / 2014$ & Misr 3 x Cross 1907 \\
\hline G10 & $2107 / 416 / 2014$ & Nubaria 3 x Cross 1906 & G40 & $2115 / 482 / 2014$ & Misr 3 x Cross 1907 \\
\hline G11 & $2107 / 420 / 2014$ & Nubaria 3 x Cross 1906 & G41 & $2115 / 483 / 2014$ & Misr 3 x Cross 1907 \\
\hline G12 & $2107 / 421 / 2014$ & Nubaria 3 x Cross 1906 & G42 & $($ Giza $3 x(837 / 461 / 83)) \times$ Cairo 25 & Cross 943 x Cairo 25 \\
\hline G13 & $2107 / 422 / 2014$ & Nubaria 3 x Cross 1906 & G43 & $($ Giza $3 x(837 / 461 / 83)) \times$ Cairo 25 & Cross 943 x Cairo 25 \\
\hline G14 & $2107 / 425 / 2014$ & Nubaria 3 x Cross 1906 & G44 & $($ Giza $3 x(837 / 461 / 83)) \times$ Cross 1906 & Cross 943 x Cross 1906 \\
\hline G15 & $2108 / 433 / 2014$ & Nubaria 3 x Cross 1907 & G45 & $($ Giza $3 x(837 / 461 / 83)) \times$ Cross 1906 & Cross 943 x Cross 1906 \\
\hline G16 & $2018 / 434 / 2014$ & Nubaria 3 x Cross 1907 & G46 & $($ Giza $3 x(837 / 461 / 83)) \times$ Cross 1906 & Cross 943 x Cross 1906 \\
\hline G17 & $2110 / 437 / 2014$ & Misr 3 x Cairo 4 & G47 & $($ Giza $3 \times(837 / 461 / 83)) \times$ Cross 1906 & Cross 943 x Cross 1906 \\
\hline G18 & $2110 / 440 / 2014$ & Misr 3 x Cairo 4 & G48 & $($ Giza $3 x(837 / 461 / 83)) \times$ Cross 1907 & Cross 943 x Cross 1907 \\
\hline G19 & $2110 / 441 / 2014$ & Misr $3 \times$ Cairo 4 & G49 & $2120 / 501 / 2014$ & Cross 943 x Cross 1907 \\
\hline G20 & $2110 / 442 / 2014$ & Misr 3 x Cairo 4 & G50 & $2121 / 503 / 2014$ & Cairo 4 x Cairo 5 \\
\hline G21 & $2110 / 443 / 2014$ & Misr 3 x Cairo 4 & G51 & $2121 / 504 / 2014$ & Cairo 4 x Cairo 5 \\
\hline G22 & $2110 / 444 / 2014$ & Misr 3 x Cairo 4 & G52 & $2123 / 505 / 2014$ & Cairo 4 x Cross 1714 \\
\hline G23 & $2110 / 450 / 2014$ & Misr 3 x Cairo 4 & G53 & $2123 / 512 / 2014$ & Cairo 4 x Cross 1714 \\
\hline G24 & $2112 / 451 / 2014$ & Misr 3 x Cairo 25 & G54 & $2124 / 513 / 2014$ & Cairo 4 x Cross 1906 \\
\hline G25 & $2112 / 452 / 2014$ & Misr $3 \times$ Cairo 25 & G55 & $2124 / 522 / 2014$ & Cairo 4 x Cross 1906 \\
\hline G26 & $2113 / 454 / 2014$ & Misr 3 x Cross 1714 & G56 & Sids 16 & $\begin{array}{l}(\text { Giza40x Misr2 }) \times(\text { Giza } 716 \times \\
\text { T.W) }\end{array}$ \\
\hline G27 & $2114 / 455 / 2014$ & Misr 3 x Cross 1906 & 57 & Giza 429 & Selection from Giza 402 \\
\hline G28 & $2114 / 459 / 2014$ & Misr 3 x Cross 1906 & 58 & Misr 3 & Misr 1 x Cairo 1 \\
\hline G29 & $2114 / 465 / 2014$ & Misr 3 x Cross 1906 & 59 & Sakha 1 & $716 / 724 / 88 \times 620 / 283 / 85$ \\
\hline G30 & $2114 / 468 / 2014$ & Misr 3 x Cross 1906 & 60 & Giza 843 & $461 / 854 / 83 \times 561 / 2076 / 85$ \\
\hline
\end{tabular}

Table 3: Field layout of alpha lattice design with 60 genotypes in 2 complete replications. Each replicate contained 5 blocks $(B)$ and each row contained 12 genotypes (G).

\begin{tabular}{|c|c|c|c|c|c|c|c|c|c|c|c|c|c|c|}
\hline \multirow[t]{2}{*}{ Rep. } & \multirow{2}{*}{$\begin{array}{c}\text { Block } \\
\text { No. } \\
\end{array}$} & \multirow{2}{*}{$\begin{array}{c}\text { Row } \\
\text { No. }\end{array}$} & \multicolumn{12}{|c|}{ G. No. } \\
\hline & & & 1 & 2 & 3 & 4 & 5 & 6 & 7 & 8 & 9 & 10 & 11 & 12 \\
\hline \multirow[t]{5}{*}{1} & 1 & 1 & 12 & 7 & 11 & 38 & 8 & 2 & 32 & 24 & 50 & 9 & 46 & 54 \\
\hline & 2 & 2 & 49 & 45 & 42 & 27 & 22 & 59 & 31 & 41 & 18 & 36 & 19 & 26 \\
\hline & 3 & 3 & 25 & 29 & 23 & 51 & 48 & 53 & 43 & 15 & 55 & 4 & 47 & 37 \\
\hline & 4 & 4 & 21 & 40 & 17 & 35 & 33 & 34 & 16 & 60 & 30 & 52 & 28 & 14 \\
\hline & 5 & 5 & 57 & 20 & 39 & 5 & 56 & 6 & 3 & 13 & 58 & 1 & 10 & 44 \\
\hline \multirow[t]{5}{*}{2} & 1 & 1 & 12 & 22 & 16 & 30 & 28 & 23 & 58 & 56 & 36 & 29 & 6 & 2 \\
\hline & 2 & 2 & 42 & 60 & 13 & 46 & 55 & 59 & 34 & 49 & 37 & 50 & 20 & 8 \\
\hline & 3 & 3 & 1 & 53 & 47 & 19 & 38 & 40 & 31 & 57 & 11 & 33 & 48 & 14 \\
\hline & 4 & 4 & 24 & 10 & 4 & 52 & 18 & 43 & 21 & 5 & 39 & 32 & 26 & 54 \\
\hline & 5 & 5 & 44 & 7 & 3 & 41 & 27 & 17 & 15 & 9 & 35 & 25 & 51 & 45 \\
\hline
\end{tabular}

\section{RESULTS AND DISCUSSION}

\section{Analysis of variance}

The analysis of variance at Sids and Nubaria Locations for faba bean yield and yield components (combined data of two years) were given in Tables (4 and 5). Results revealed that, the mean squares of genotypes were highly significant $(p \leq 0.01)$ for all studied traits in both locations, exceptfor, number of branches.plant ${ }^{-1}$ in Sids and number of pods. Plant ${ }^{-1}$ in Nubaria. These results a considerable genetic variation in those materials, 
indicate might results were these in agreement with those obtained by Zarea-Fizabady and Ghodsi (2004), Abd El-Mohsen and Abo-Hegazy (2013), Abd El-Shafi (2014) and Mona et al. (2018).

Mean performance of genotypes at Sids and Nubaria locations for faba bean, yield and yield components were presented in Tables (6 and 7). The results indicated that, the highest values of plant height were obtained from genotype G50 followed by G5 and G53with averages of 145.0, 142.5 and $141.5 \mathrm{~cm}$ in Sids location, respectively. While in Nubaria location, the highest values were obtained from genotypes G2 followed by G1 and G39 with averages of $123,115.8$ and $112.6 \mathrm{~cm}$., respectively. Regarding to the number of branches. plant ${ }^{-1}$, in Sids location the values were ranged from 3.48 to 5.73 (G6 or G13). While at Nubaria location, the values ranged from $2.67(\mathrm{G} 2)$ to 6.21 (G14). On the other hand, the genotype G42 and G11 gave the highest value for number of pods.plant ${ }^{-1}$ as 37.52 and 18.55 in Sids and Nubaria location, respectively. Data for number of seed.plant ${ }^{-1}$ showed that, genotypes G42, G43 and G22 recorded 120.77, 102.17 and 101.95 respectively, in Sids location, whereas, in Nubaria location genotypes G46, G44 and G50 scored $62.28,60.88$ and 58.54, respectively. In addition, G13 had the highest value of 100- seed weight followed by G40 and G47 with average of 101.8 and $99.86(\mathrm{~g})$, respectively in Sids location, while in Nubaria location, genotypes G51 followed by G10 and G8 recoded of 88.93, 85.98 and $84.3 \mathrm{~g}$, respectively (Table 6 and 7). These results are in agreement with those obtained bySilim and Saxena, (1992) and Al Ghamdi, (2007).

The highest value of seed weight.plant ${ }^{-1}$ was recorded by the genotype G42 (98.55 g), followed by genotype G1 (89.1 g) and G22 (88.8g), while the lowest values were exhibited by cultivar Miser3 (19.75 g), Giza 429 (26.55 g) and genotype G32 (33.9 g) in Sids location. Whereas at Nubaria location the seed weight.plant ${ }^{-1}$ ranged between $50.0 \mathrm{~g}$ for genotype $\mathrm{G} 44$ to $21.47 \mathrm{~g}$ for genotype G15.

Regarding faba bean seed yield, the highest value was produced by genotype G27 (15.57 $\left.\operatorname{ardab} \mathrm{fad}^{-1}\right)$ followed by genotype G53 (15.1 $\left.\operatorname{ardab} . \mathrm{fad}^{-1}\right)$ and genotype G52 (14.58 $\left.\operatorname{ardab} \mathrm{fad}^{-1}\right)$, while the least seed yield was produced by genotype G23 (9.68 ardab.fad $^{-1}$ ) in Sids region. On the other hand, in Nubaria location, the highest value of seed yield given by genotype $\mathrm{G} 45$ followed by genotype G55 and G44 (11.63, 10.74 and $10.5 \mathrm{~kg}$, respectively), while the least seed yield was produced by genotype G15 (4.58 $\left.\operatorname{ardab} \mathrm{fad}^{-1}\right)$

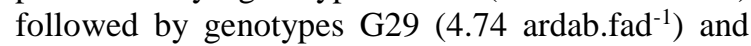
$\mathrm{G} 21(4.95 \mathrm{~kg})$. These results are in accordance with those obtained by (1991, Al-Rifaee, 1999 ,and Rehab, 2014).

Table 4: Mean squares of 60 faba bean genotypes (yield and yield components combined over two years) at Sids location.

\begin{tabular}{lcccccccc}
\hline S.O.V & df & $\begin{array}{c}\text { Plant } \\
\text { height } \\
\text { cm }\end{array}$ & $\begin{array}{c}\text { No. of } \\
\text { branches. } \\
\text { plant }\end{array}$ & $\begin{array}{c}\text { No. of pod } \\
\text { plant }^{-1}\end{array}$ & $\begin{array}{c}\text { No. of } \\
\text { seed.Plant-1 }\end{array}$ & $\begin{array}{c}\text { seed } \\
\text { weight. } \\
\text { Plant }^{-1}(\mathbf{g})\end{array}$ & $\begin{array}{c}\text { 100-seed } \\
\text { weight(g) }\end{array}$ & $\begin{array}{c}\text { Seed } \\
\text { Yield } \\
\text { ard.fad }^{-1}\end{array}$ \\
\hline Year & 1 & $966.5^{* *}$ & 3.15 & $6274.00^{* *}$ & $1966^{* *}$ & 40.44 & 5117.36 & 0.3 \\
\hline Year*rep & 2 & 338.1 & $10.05^{* *}$ & $391.38^{* *}$ & $138.7^{* *}$ & 63.46 & 334.51 & 23.46 \\
\hline Year*rep*blok & 20 & $252.4^{* *}$ & 1.17 & 71.83 & $252.7^{* *}$ & $236.16^{* *}$ & 79.52 & $3.66^{* *}$ \\
\hline Genotypes & 59 & $333.4^{* *}$ & 1.27 & $142.66^{* *}$ & $362.2^{* *}$ & $485.92^{* *}$ & $175.84 * *$ & $5.85^{* *}$ \\
\hline Year*G & 59 & $223.6^{* *}$ & 1.51 & $91.31^{* *}$ & $223.6^{* *}$ & $364.51^{* *}$ & 99.13 & $3.17^{* *}$ \\
\hline Residual & 98 & 129.7 & 1.17 & 48.83 & 129.1 & 95.73 & 59.45 & 4.53 \\
\hline
\end{tabular}

*,** = Significant at 1 and 5\% probability level, respectively.

Table 5; Mean squares of 60 faba bean genotypes (yield and yield components) (combined over two years) at Nubaria location.

\begin{tabular}{lcccccccc}
\hline S.O.V & df & $\begin{array}{c}\text { Plant } \\
\text { height } \\
\text { cm }\end{array}$ & $\begin{array}{c}\text { No. of } \\
\text { branches. } \\
\text { plant }^{-1}\end{array}$ & $\begin{array}{c}\text { No. of } \\
\text { pod } \\
\text {.plant }^{-1}\end{array}$ & $\begin{array}{c}\text { No. of } \\
\text { seed.Plant-1 }\end{array}$ & $\begin{array}{c}\text { seed } \\
\text { weight. } \\
\text { Plant }^{-1}(\mathbf{g})\end{array}$ & $\begin{array}{c}\text { 100-seed } \\
\text { weight(g) }\end{array}$ & $\begin{array}{c}\text { Seed } \\
\text { Yield } \\
\text { ard.fad }^{-\mathbf{1}}\end{array}$ \\
\hline Year & 1 & 606.74 & $60.669^{* *}$ & 362.3 & $9154.36^{* *}$ & 3.63 & 34881.8 & 2.058 \\
\hline Year/ rep & 2 & 111.65 & $41.945^{* *}$ & 1.9 & $729.15^{* *}$ & 26.57 & 1422.5 & 14.754 \\
\hline rep*block/ Year & 20 & $84.65^{* *}$ & $3.01^{*}$ & 8.17 & 123.97 & $34.43^{* *}$ & 546.7 & 1.62 \\
\hline Genotypes & 59 & $112.18^{* *}$ & $3.47 *$ & 17.95 & $212.61 * *$ & $136.93 * *$ & $871.1 * *$ & $8.53^{* *}$ \\
\hline Year*G & 59 & $112.56^{* *}$ & 1.91 & 20.5 & 132.34 & $35.168^{* *}$ & 428.7 & 1.42 \\
\hline Residual & 98 & 61.46 & $2.62^{*}$ & 15.69 & 99.6 & 8.92 & 48.8 & 6.25 \\
\hline
\end{tabular}

$*, * *=$ Significant at 1 and $5 \%$ probability level, respectively. 
Table 6: Means of plant height, number of branches, pods and seeds. plant ${ }^{-1}$ for sixty faba bean genotypes at Sids location.

\begin{tabular}{|c|c|c|c|c|c|c|c|c|c|}
\hline Genotypes & $\begin{array}{c}\text { Plant } \\
\text { height } \\
\text { cm }\end{array}$ & $\begin{array}{c}\text { No. of } \\
\text { branches. } \\
\text { Plant }^{-1}\end{array}$ & $\begin{array}{l}\text { No. of } \\
\text { pods. } \\
\text { Plant }^{-1} \\
\end{array}$ & $\begin{array}{c}\text { No. of } \\
\text { seed. } \\
\text { plant }^{-1} \\
\end{array}$ & Genotypes & $\begin{array}{c}\text { Plant } \\
\text { height } \\
\text { cm } \\
\end{array}$ & $\begin{array}{c}\text { No. of } \\
\text { branches. } \\
\text { Plant }^{-1} \\
\end{array}$ & $\begin{array}{c}\text { No. of } \\
\text { pods.Plant }^{-1}\end{array}$ & $\begin{array}{c}\text { No. of } \\
\text { seed. } \\
\text { Plant }\end{array}$ \\
\hline G1 & 127.50 & 4.08 & 22.48 & 64.45 & G31 & 124.50 & 5.63 & 17.72 & 54.27 \\
\hline G2 & 130.00 & 4.53 & 21.72 & 65.23 & G32 & 132.50 & 4.85 & 11.52 & 35.72 \\
\hline G3 & 128.70 & 4.90 & 21.72 & 55.9 & G33 & 132.50 & 4.60 & 18.27 & 55.3 \\
\hline G4 & 117.50 & 5.48 & 24.4 & 74.28 & G34 & 134.50 & 4.43 & 21.47 & 66.15 \\
\hline G5 & 117.50 & 4.83 & 20.47 & 65.4 & G35 & 133.70 & 4.98 & 21.72 & 70.98 \\
\hline G6 & 126.20 & 5.73 & 29.52 & 84.35 & G36 & 127.50 & 4.83 & 20.07 & 58.02 \\
\hline G7 & 117.50 & 5.23 & 30.77 & 98.82 & G37 & 132.50 & 4.95 & 30.85 & 88.52 \\
\hline G8 & 107.50 & 4.98 & 20.60 & 68.58 & G38 & 116.20 & 4.73 & 34.45 & 91.2 \\
\hline G9 & 110.00 & 5.65 & 23.90 & 71.2 & G39 & 135.00 & 5.70 & 22.4 & 67.1 \\
\hline G10 & 110.00 & 5.08 & 15.95 & 52.85 & G40 & 140.00 & 4.83 & 26.52 & 81.6 \\
\hline G11 & 120.00 & 5.65 & 16.02 & 54.77 & G41 & 127.50 & 5.35 & 32.10 & 97.65 \\
\hline G12 & 102.50 & 4.23 & 19.27 & 59.98 & G42 & 125.00 & 4.53 & 37.52 & 120.77 \\
\hline G13 & 125.00 & 5.73 & 25.65 & 69.85 & G43 & 139.50 & 5.70 & 28.9 & 102.17 \\
\hline G14 & 135.00 & 4.18 & 29.65 & 86.70 & G44 & 103.70 & 4.28 & 12.6 & 50.58 \\
\hline G15 & 111.20 & 4.65 & 27.4 & 64.43 & G45 & 137.50 & 4.30 & 30.17 & 86.12 \\
\hline G16 & 122.50 & 4.55 & 26.4 & 75.62 & G46 & 132.50 & 5.63 & 28.00 & 88.97 \\
\hline G17 & 125.00 & 4.90 & 25.2 & 84.72 & G47 & 131.20 & 5.53 & 28.15 & 83.22 \\
\hline G18 & 115.00 & 4.45 & 18.47 & 68.47 & G48 & 135.00 & 5.38 & 22.8 & 73.53 \\
\hline G19 & 132.50 & 4.35 & 23.77 & 78.65 & G49 & 130.00 & 4.53 & 32.07 & 95.2 \\
\hline $\mathrm{G} 20$ & 132.50 & 4.40 & 22.27 & 60.53 & G50 & 145.00 & 4.25 & 22.77 & 77.9 \\
\hline G21 & 126.20 & 3.83 & 19.97 & 59.08 & G51 & 142.50 & 4.15 & 24.47 & 68.9 \\
\hline G22 & 125.00 & 4.65 & 31.4 & 101.95 & G52 & 138.70 & 4.03 & 24.07 & 71.75 \\
\hline $\mathrm{G} 23$ & 122.50 & 3.98 & 17.02 & 58.15 & G53 & 141.50 & 4.33 & 28.02 & 83.82 \\
\hline G24 & 128.70 & 3.48 & 18.65 & 64.45 & G54 & 130.00 & 4.78 & 31.7 & 91.07 \\
\hline G25 & 125.00 & 5.45 & 20.47 & 60.00 & G55 & 130.00 & 4.78 & 19.35 & 56.03 \\
\hline G26 & 117.50 & 4.28 & 17.9 & 60.17 & G 56 & 125.2 & 4.44 & 26.43 & 77.95 \\
\hline G27 & 127.50 & 4.15 & 14.05 & 50.35 & Giza 843 & 120.6 & 5.14 & 21.01 & 77.92 \\
\hline G28 & 130.00 & 3.78 & 14.97 & 49.95 & Giza 429 & 122.5 & 4.75 & 11.5 & 33.5 \\
\hline G29 & 118.70 & 4.65 & 16.5 & 54.33 & Misr 3 & 100 & 4.5 & 11.5 & 29.75 \\
\hline G30 & 135.00 & 4.03 & 20.15 & 53.27 & Sakha 1 & 127.3 & 4.98 & 21.74 & 64.48 \\
\hline
\end{tabular}

Table 7: Means of seed weight. Plant $^{-1}, 100$-seed weight and seed yield for sixty faba bean genotypes at Sids location.

\begin{tabular}{|c|c|c|c|c|c|c|c|}
\hline Genotypes & $\begin{array}{l}\text { Seed weight. } \\
\text { Plant }^{-1} \\
\text { (g) }\end{array}$ & $\begin{array}{l}\text { 100-seed } \\
\text { weight } \\
\text { (g) }\end{array}$ & $\begin{array}{c}\text { seed yield } \\
\text { ard.fad }^{-1}\end{array}$ & Genotypes & $\begin{array}{l}\text { Seed weight. } \\
\text { Plant }^{-1} \\
\text { (g) }\end{array}$ & $\begin{array}{l}\text { 100-seed } \\
\text { weight } \\
\text { (g) }\end{array}$ & $\begin{array}{c}\text { seed } \\
\text { yield } \\
\text { ard.fad }^{-1}\end{array}$ \\
\hline G1 & 50.05 & 73.40 & 13.59 & G31 & 43.03 & 79.23 & 13.69 \\
\hline $\mathrm{G} 2$ & 46.60 & 68.65 & 11.37 & G32 & 33.90 & 94.55 & 13.10 \\
\hline G3 & 41.82 & 74.65 & 8.92 & G33 & 43.35 & 78.69 & 12.72 \\
\hline G4 & 54.40 & 73.50 & 11.34 & G34 & 60.72 & 92.06 & 13.47 \\
\hline G5 & 40.15 & 60.63 & 12.24 & G35 & 66.15 & 92.45 & 13.73 \\
\hline G6 & 62.70 & 75.54 & 11.29 & G36 & 62.12 & 97.82 & 12.35 \\
\hline G7 & 78.08 & 78.28 & 11.96 & G37 & 71.27 & 80.16 & 12.77 \\
\hline G8 & 60.78 & 91.46 & 11.34 & G38 & 83.35 & 91.73 & 11.48 \\
\hline G9 & 60.97 & 84.98 & 12.97 & G39 & 54.80 & 83.75 & 12.35 \\
\hline G10 & 47.65 & 90.37 & 11.24 & G40 & 89.10 & 101.18 & 13.22 \\
\hline G11 & 44.97 & 86.02 & 12.52 & G41 & 70.55 & 73.2 & 13.38 \\
\hline G12 & 49.45 & 81.89 & 10.97 & G42 & 98.55 & 94.8 & 12.10 \\
\hline G13 & 71.08 & 101.6 & 11.38 & G43 & 76.90 & 74.75 & 12.92 \\
\hline G14 & 73.65 & 84.33 & 11.17 & G44 & 37.52 & 72.71 & 13.22 \\
\hline G15 & 47.17 & 78.86 & 11.32 & G45 & 67.70 & 84.22 & 12.31 \\
\hline
\end{tabular}


Cont. Table 7: Means of seed weight. Plant $^{-1}$, 100-seed weight and seed yield for sixty faba bean genotypes at Sids location.

\begin{tabular}{|c|c|c|c|c|c|c|c|}
\hline Genotypes & $\begin{array}{l}\text { Seed weight. } \\
\text { Plant }^{-1} \\
(\mathrm{~g})\end{array}$ & $\begin{array}{l}\text { 100-seed } \\
\text { weight } \\
\text { (g) }\end{array}$ & $\begin{array}{l}\text { seed yield } \\
\text { ard.fad }^{-1}\end{array}$ & Genotypes & $\begin{array}{l}\text { Seed weight. } \\
\text { Plant }^{-1} \\
\text { (g) }\end{array}$ & $\begin{array}{l}\text { 100-seed } \\
\text { weight } \\
\text { (g) }\end{array}$ & $\begin{array}{c}\text { seed yield } \\
\text { ard.fad }^{-1}\end{array}$ \\
\hline G16 & 69.82 & 90.10 & 10.20 & G46 & 67.77 & 79.49 & 12.32 \\
\hline G17 & 70.27 & 81.49 & 13.16 & G47 & 83.45 & 99.86 & 11.97 \\
\hline G18 & 58.40 & 85.79 & 11.12 & G48 & 48.30 & 65.56 & 11.75 \\
\hline G19 & 62.90 & 78.64 & 13.06 & G49 & 84.90 & 86.56 & 12.13 \\
\hline G20 & 54.90 & 89.87 & 12.89 & G50 & 63.32 & 80.76 & 11.66 \\
\hline $\mathrm{G} 21$ & 47.03 & 78.00 & 12.04 & G51 & 56.87 & 82.10 & 11.48 \\
\hline $\mathrm{G} 22$ & 88.80 & 87.13 & 13.02 & G52 & 53.97 & 74.83 & 14.58 \\
\hline G23 & 52.60 & 87.33 & 9.68 & G53 & 61.80 & 73.58 & 15.10 \\
\hline G24 & 54.15 & 92.85 & 13.60 & G54 & 73.37 & 80.42 & 12.79 \\
\hline $\mathrm{G} 25$ & 52.22 & 88.48 & 11.88 & G55 & 43.15 & 75.32 & 10.96 \\
\hline G26 & 49.60 & 83.67 & 12.10 & G 56 & 66.79 & 83.68 & 11.06 \\
\hline G27 & 44.80 & 89.38 & 15.57 & Giza 843 & 64.23 & 83.03 & 12.19 \\
\hline $\mathrm{G} 28$ & 41.83 & 83.73 & 12.42 & Giza 429 & 26.25 & 78.34 & 12.78 \\
\hline $\mathrm{G} 29$ & 49.45 & 93.57 & 12.30 & Misr 3 & 19.75 & 66.19 & 10.62 \\
\hline G30 & 47.55 & 88.68 & 13.05 & Sakha 1 & 58.14 & 89.1 & 12.56 \\
\hline
\end{tabular}

Table 8: Means of Plant height, number of branches, pods and seeds.plant ${ }^{-1}$ for sixty faba bean genotypes at Nubaria location

\begin{tabular}{|c|c|c|c|c|c|c|c|c|c|}
\hline Genotypes & $\begin{array}{c}\text { Plant } \\
\text { height } \\
\text { cm } \\
\end{array}$ & $\begin{array}{c}\text { No. of } \\
\text { branches. } \\
\text { Plant }^{-1} \\
\end{array}$ & $\begin{array}{c}\text { No. of } \\
\text { pods. } \\
\text { Plant }^{-1} \\
\end{array}$ & $\begin{array}{c}\text { No. of } \\
\text { seed. } \\
\text { plant }^{-1} \\
\end{array}$ & Genotypes & $\begin{array}{c}\text { Plant } \\
\text { height } \\
\text { cm } \\
\end{array}$ & $\begin{array}{c}\text { No. of } \\
\text { branches. } \\
\text { Plant }^{-1} \\
\end{array}$ & $\begin{array}{l}\text { No. of } \\
\text { pods. } \\
\text { Plant }^{-1} \\
\end{array}$ & $\begin{array}{c}\text { No. of } \\
\text { seed. } \\
\text { plant }^{-1}\end{array}$ \\
\hline G1 & 115.80 & 4.75 & 12.66 & 44.34 & G31 & 102.10 & 5.17 & 15.16 & 102.10 \\
\hline G2 & 123.00 & 2.67 & 11.19 & 45.69 & G32 & 106.40 & 5.75 & 14.23 & 44.62 \\
\hline G3 & 105.70 & 3.25 & 13.79 & 45.36 & G33 & 104.00 & 4.38 & 14.52 & 43.42 \\
\hline G4 & 110.90 & 3.08 & 14.46 & 45.23 & G34 & 102.80 & 4.50 & 13.78 & 41.89 \\
\hline G5 & 100.90 & 3.96 & 12.23 & 38.33 & G35 & 100.40 & 4.13 & 13.47 & 47.33 \\
\hline G6 & 111.50 & 4.63 & 14.64 & 49.65 & G36 & 98.40 & 4.79 & 16.50 & 39.69 \\
\hline G7 & 103.10 & 4.67 & 14.89 & 53.29 & G37 & 102.10 & 5.58 & 13.72 & 45.44 \\
\hline G8 & 105.00 & 5.25 & 13.32 & 33.78 & G38 & 100.00 & 4.13 & 13.57 & 41.06 \\
\hline G9 & 104.90 & 5.42 & 11.23 & 34.90 & G39 & 112.60 & 4.00 & 13.58 & 44.91 \\
\hline G10 & 102.10 & 4.33 & 16.75 & 46.83 & G40 & 101.60 & 3.88 & 12.24 & 44.03 \\
\hline G11 & 102.70 & 3.67 & 18.55 & 42.88 & G41 & 98.60 & 5.71 & 13.37 & 46.72 \\
\hline G12 & 101.60 & 2.75 & 14.95 & 42.04 & G42 & 109.80 & 5.08 & 14.79 & 50.98 \\
\hline G13 & 99.70 & 5.63 & 12.54 & 37.48 & G43 & 101.00 & 5.29 & 12.72 & 52.38 \\
\hline G14 & 97.90 & 6.21 & 12.95 & 32.20 & G44 & 107.10 & 4.33 & 17.74 & 60.88 \\
\hline G15 & 96.50 & 4.96 & 9.77 & 26.69 & G45 & 107.40 & 5.50 & 14.93 & 53.23 \\
\hline G16 & 97.40 & 4.63 & 12.48 & 36.41 & G46 & 115.30 & 4.92 & 15.72 & 62.28 \\
\hline G17 & 106.20 & 3.79 & 13.65 & 40.83 & G47 & 104.40 & 4.25 & 14.58 & 40.74 \\
\hline G18 & 110.30 & 4.71 & 18.36 & 50.53 & G48 & 109.70 & 3.58 & 15.77 & 58.18 \\
\hline G19 & 110.90 & 5.42 & 15.97 & 46.34 & G49 & 104.80 & 4.13 & 12.92 & 40.19 \\
\hline G20 & 110.40 & 5.38 & 11.58 & 40.48 & G50 & 108.20 & 2.83 & 17.42 & 58.54 \\
\hline G21 & 108.30 & 4.21 & 10.82 & 41.61 & G51 & 95.30 & 3.00 & 13.48 & 42.93 \\
\hline G22 & 106.20 & 4.79 & 14.56 & 40.28 & G52 & 105.40 & 3.21 & 15.28 & 44.00 \\
\hline G23 & 107.00 & 4.42 & 16.34 & 48.89 & G53 & 103.70 & 3.13 & 13.85 & 43.77 \\
\hline G24 & 92.70 & 5.33 & 12.89 & 42.26 & G54 & 110.10 & 2.92 & 12.48 & 44.33 \\
\hline G25 & 105.40 & 5.38 & 10.70 & 37.45 & G55 & 110.80 & 3.00 & 10.88 & 36.28 \\
\hline G26 & 106.70 & 3.08 & 11.66 & 32.29 & G 56 & 99.2 & 3.83 & 12.15 & 38.79 \\
\hline G27 & 104.70 & 4.50 & 13.64 & 38.04 & Giza 843 & 101 & 3.25 & 12.98 & 39.48 \\
\hline G28 & 107.80 & 4.25 & 10.24 & 28.14 & Giza 429 & 101.6 & 2.92 & 9.82 & 35.87 \\
\hline G29 & 93.00 & 4.54 & 14.57 & 50.13 & Misr 3 & 105 & 2.96 & 15.66 & 50.85 \\
\hline G30 & 104.00 & 5.38 & 11.41 & 34.00 & Sakha 1 & 105 & 4.63 & 15.26 & 50.28 \\
\hline
\end{tabular}

L.S.D 0.05 Plant height: 5.54, No. of branches.Plant ${ }^{-1}: 1.14$ and No. of seed.plant ${ }^{-1}: 7.06$ 
Table 9: Means of seed weight. Plant ${ }^{-1}, 100$-seed weight and seed yield for sixty faba bean genotypes at Nubaria location

\begin{tabular}{|c|c|c|c|c|c|c|c|}
\hline Genotypes & $\begin{array}{l}\text { Seed weight. } \\
\text { Plant }^{-1} \\
\text { (g) }\end{array}$ & $\begin{array}{c}\text { 100-seed } \\
\text { weight } \\
(\mathrm{g})\end{array}$ & $\begin{array}{l}\text { seed yield } \\
\operatorname{ard.fad}^{-1}\end{array}$ & Genotypes & $\begin{array}{l}\text { Seed weight. } \\
\text { Plant }^{-1} \\
\text { (g) }\end{array}$ & $\begin{array}{c}\text { 100-seed } \\
\text { weight } \\
\text { (g) }\end{array}$ & $\begin{array}{c}\text { seed yield } \\
\text { ard.fad }^{-1}\end{array}$ \\
\hline G1 & 40.95 & 83.57 & 8.44 & G31 & 28.98 & 74.09 & 6.55 \\
\hline $\mathrm{G} 2$ & 36.87 & 80.52 & 7.46 & $\mathrm{G} 32$ & 32.74 & 72.79 & 6.42 \\
\hline G3 & 34.08 & 74.98 & 7.50 & G33 & 32.25 & 76.99 & 6.88 \\
\hline G4 & 31.19 & 70.92 & 6.78 & G34 & 26.05 & 62.77 & 7.64 \\
\hline G5 & 27.76 & 73.92 & 5.86 & G35 & 34.28 & 72.3 & 6.51 \\
\hline G6 & 38.92 & 78.77 & 7.19 & G36 & 30.68 & 77.17 & 6.13 \\
\hline G7 & 44.20 & 83.15 & 6.30 & G37 & 33.95 & 74.72 & 7.17 \\
\hline G8 & 27.95 & 84.31 & 7.48 & $\mathrm{G} 38$ & 28.51 & 71.09 & 6.17 \\
\hline G9 & 27.57 & 79.29 & 6.47 & G39 & 32.32 & 71.74 & 6.69 \\
\hline G10 & 40.52 & 85.98 & 7.05 & G40 & 36.29 & 82.54 & 5.88 \\
\hline G11 & 34.53 & 80.30 & 8.34 & G41 & 38.69 & 82.86 & 9.01 \\
\hline G12 & 34.86 & 82.05 & 6.62 & G42 & 41.34 & 81.32 & 7.57 \\
\hline G13 & 30.59 & 82.89 & 6.45 & G43 & 43.53 & 83.23 & 7.70 \\
\hline G14 & 26.54 & 73.35 & 7.63 & G44 & 50.00 & 82.01 & 10.57 \\
\hline G15 & 21.47 & 78.48 & 4.58 & G45 & 43.44 & 82.70 & 11.63 \\
\hline G16 & 26.61 & 73.03 & 6.58 & G46 & 47.86 & 76.67 & 7.53 \\
\hline G17 & 32.95 & 79.63 & 7.04 & G47 & 34.06 & 81.10 & 6.36 \\
\hline G18 & 36.57 & 72.44 & 7.03 & G48 & 45.07 & 77.05 & 9.36 \\
\hline G19 & 33.37 & 72.22 & 6.43 & G49 & 31.33 & 77.89 & 8.93 \\
\hline G20 & 30.40 & 75.26 & 5.53 & G50 & 48.42 & 82.36 & 10.41 \\
\hline $\mathrm{G} 21$ & 28.25 & 69.38 & 4.95 & G51 & 38.14 & 88.93 & 6.03 \\
\hline G22 & 28.45 & 71.63 & 6.14 & G52 & 36.52 & 82.90 & 7.83 \\
\hline G23 & 36.71 & 74.34 & 7.96 & G53 & 38.19 & 87.37 & 7.67 \\
\hline G24 & 31.83 & 74.84 & 5.91 & G54 & 28.27 & 65.76 & 6.75 \\
\hline G25 & 26.88 & 72.68 & 6.12 & G55 & 28.17 & 77.36 & 10.74 \\
\hline G26 & 25.44 & 77.60 & 8.07 & G 56 & 29.02 & 75.03 & 5.62 \\
\hline G27 & 27.90 & 74.61 & 6.36 & Giza 843 & 29.47 & 73.13 & 5.91 \\
\hline G28 & 18.42 & 65.87 & 5.86 & Giza 429 & 24.96 & 69.64 & 8.44 \\
\hline G29 & 37.96 & 77.01 & 4.74 & Misr 3 & 32.64 & 67.34 & 6.78 \\
\hline G30 & 28.57 & 79.59 & 7.24 & Sakha 1 & 39.42 & 77.56 & 8.00 \\
\hline
\end{tabular}

L.S.D 0.05 Seed weight.Plant-1: $5.45,100$-seed weight: 2.05 and seed yield: 1.77

\section{Cluster analysis:}

Cluster analysis creates groups of samples based on their distances. It seeks objects groups of the same properties which differ at the same time from the next objects group.

The genetic euclidean distances among 60 genotypes derived from the two locations (Sids and Nubaria) were aggregated into phenotypic groups. The cluster analysis was used as an efficient procedure to emerge the structural relationships among tested genotypes and provides a hierarchical classification of them.

In the present work, the similarity levels of the two locations were estimated based on seed yield plant ${ }^{-1}$ and its related characters. Such genotypes were separated into meaningful genetic division based on knowledge of pedigree and type of these genotypes (Table 10). A dendrogram was constructed on the basis of co-ordinates of principal component analysis. Cluster group rankings were obtained based on Ward's minimum variance. Phylogenetic tree, in figures (1 and 2). The clustering pattern of genotypes derived from sids showed four kinds of grouping. These four clusters consisted of $15, \quad 15, \quad 10$ and 20 members, respectively (Fig. 1) Clusters I, II, III and IV have the similarity level $7.18, \quad 10.42,4.12$ and 9.38, respectively. The cluster (I) aggregated the genotypes that had the highest plant height ${ }^{-1}$ and seed yield. 
While the third (III) cluster had the No. of branches and seed weight plants ${ }^{-1}$ but the fourth had the highest No. of pods, seeds plant $^{-1}$ and 100 -seed weight. So that, the first cluster (I) aggregated the genotypes that had the highest seed yield ardab.fad ${ }^{-1}$ followed by third cluster (III). The dendogram of population derived from the Nubaria consisted of four clusters. The genotypes were classified in each cluster presented 16, 12, 15 and $17 \%$ of total genotypes respectively (Fig. 2). The first cluster (I) was for the highest plant height, No. of pods plant ${ }^{-1}$, 100-seed weight and seed yield $\left(\operatorname{ardab} . \mathrm{fad}^{-1}\right)$. By contrast, the second cluster
(II) aggregated the genotypes that had the highest seed weight plant $^{-1}$. Also the fourth cluster aggregated the plant that had the highest No. of branches plants ${ }^{-1}$. and seed yield plant $^{-1}$. So that, the first cluster possessed the highest estimates of most studied traits compared with the other clusters followed by the second cluster (II) (Table 11). The third cluster content most commercial cultivars. Cluster analysis provided with a complete view of the variation present among the 60 faba bean. Genotypes and it might be used by plant breeders during genetic improvement of faba bean.

Table 10: Elite genotypes of the studied traits according to the alpha lattice design in the two locations.

\begin{tabular}{|c|c|c|}
\hline Traits & Sids & Nubaria \\
\hline Plant height & $\begin{array}{l}\text { G1, G2, G3, G14, G19, G20, G24, } \\
\text { G27, G28, G30, G32, G33, G34, G35, } \\
\text { G36, G37, G39, G40, G41, G43, G45, } \\
\text { G46,G47,G48, G49, G50, G51, G52, } \\
\text { G53,G54, G55, G57, G58, G60, G56. }\end{array}$ & $\begin{array}{l}\text { G1, G2, G3, G4, G6, G8, G17, G18 } \\
\text { G19, G20, G21, G22, G23, G25, G26 } \\
\text { G28, G32, G42, G44, G45, G46, G48 } \\
\text { G50, G52, G54, G55, G59, G60. }\end{array}$ \\
\hline No. of branches.Plant- ${ }^{1}$ & $\begin{array}{l}\text { G4, G5, G16, G7, G8,G9, G10, G11, } \\
\text { G13, G17, G25, G31, G32, G35, G36, } \\
\text { G37, G39, G40, G43, G45, G46, G47, } \\
\text { G48, G54, G55, G57, G60. }\end{array}$ & $\begin{array}{l}\text { G1, G6, G7, G8, G9, G13, G14, } \\
\text { G15, G16, G18, G19, G 20, G22, } \\
\text { G23, G24, G25, ,G27, G29, G30, } \\
\text { G31,G32, G34, G36, G37, G41, } \\
\text { G42, G43,G45,G46, G60, G56. }\end{array}$ \\
\hline No. of pods.Plant ${ }^{-1}$ & $\begin{array}{l}\text { G6, G7,G8,G9, G13, G14,G16,G17, } \\
\text { G19, G20, G37, G38,G39, G40, G41, } \\
\text { G42, G43, G45, G46, G47, G49, G51, } \\
\text { G52, G53, G54, G57, G60, G56. }\end{array}$ & $\begin{array}{l}\text { G4, G6, G7, G10,G11,G12, G18, } \\
\text { G19, G22, G23, G29,G31, G32, } \\
\text { G33, G42, G44, G45, G46, G47, } \\
\text { G48, G50, G59, G60. }\end{array}$ \\
\hline No. of seed.Plant ${ }^{-1}$ & $\begin{array}{l}\text { G6, G7, G14,G22,G37, G38,G40, G41, } \\
\text { G42, G43, G45, G46, G47, G49, G50, } \\
\text { G53, G54,G57, G60, G56. }\end{array}$ & $\begin{array}{l}\text { G1, G2, G3, G4, G6, G7, G10, G18 } \\
\text { G19,G20, G29, G32, G35, G39, G40 } \\
\text { G41, G42, G43, G44, G45, G46, G48 } \\
\text { G50, G52, G59, G60. }\end{array}$ \\
\hline seed weight.Plant ${ }^{-1}(\mathrm{~g})$ & $\begin{array}{l}\text { G6, G7,G8,G9, G13, G14, G17, G19, } \\
\text { G22,G34, G35, G36,G37, G38, G40, } \\
\text { G41, G42, G43, G45, G46, G47, G49, } \\
\text { G50, G53, G54, G57, G60, G56. }\end{array}$ & $\begin{array}{l}\text { G1, G2, G3, G6, G7,G8, G10, } \\
\text { G11,G12, G17,G18, G23, G29, G35, } \\
\text { G37, G40, G41,G42, G43,G44, G45, } \\
\text { G46, G47,G48 G50, G51, G52, G53, } \\
\text { G59, G60. }\end{array}$ \\
\hline 100-seed weight(g) & $\begin{array}{l}\text { G8,G11, G13, G14,G16,G17, G18, } \\
\text { G20, G22, G23,G24, G25, G26, G27, } \\
\text { G28, G29,G30, G32, G34, G35, G36, } \\
\text { G38, G39, G40, G45, G47, G49, G57, } \\
\text { G60, G56. }\end{array}$ & $\begin{array}{l}\text { G1, G2, G6, G7,G8, G9, } \\
\text { G10,G11,G12, G13, G15,G17,G26, } \\
\text { G40, G41, G42, G43 ,G44, G45, } \\
\text { G47, G49, G50, G51, G52, G53, } \\
\text { G55, G57,G 60,G59. }\end{array}$ \\
\hline Seed Yield Ard.fad ${ }^{-1}$ & $\begin{array}{l}\text { G1,G9,G11, G17, G19, G20, G22, } \\
\text {,G24, G27, G28, G30, G31,G32, G33, } \\
\text { G34, G35, G36, G37, G39, G40, G41, } \\
\text { G43, G44, G46, G52, G53, G57, G58, } \\
\text { G60. }\end{array}$ & $\begin{array}{l}\text { G1, G2, G3, G6, G11,G14,G23,G26, } \\
\text { G30, G37, G41, G42, G43, G44, } \\
\text { G45, G46, G48 G50, G52, G53, } \\
\text { G55,G58, G60. }\end{array}$ \\
\hline
\end{tabular}


Table 11: Summary of cluster analysis showed the included genotypes, distance level and cluster mean of the 60 faba bean genotypes using the studied yield characters.

\begin{tabular}{|c|c|c|c|c|c|c|c|c|c|}
\hline Location & $\begin{array}{l}\text { No. of } \\
\text { genotypes } \\
\text { /Cluster }\end{array}$ & $\begin{array}{c}\text { Distance } \\
\text { level }\end{array}$ & $\begin{array}{c}\text { Plant } \\
\text { height } \\
\text { cm }\end{array}$ & $\begin{array}{c}\text { No. of } \\
\text { branches. } \\
\text { plant }^{-1}\end{array}$ & $\begin{array}{l}\text { No. of } \\
\text { pod. } \\
\text { plant }^{-1}\end{array}$ & $\begin{array}{l}\text { No. of } \\
\text { seed. } \\
\text { plant }^{-1}\end{array}$ & $\begin{array}{c}\text { seed } \\
\text { weight. } \\
\text { Plant }^{-1}(g)\end{array}$ & $\begin{array}{c}100- \\
\text { seed } \\
\text { weight }(\mathrm{g})\end{array}$ & $\begin{array}{c}\text { Seed } \\
\text { yield } \\
\text { ardab. } \\
\text { fad }^{-1}\end{array}$ \\
\hline \multirow{4}{*}{ Sids } & 15 & 7.18 & 131.72 & 4.27 & 19.53 & 59.86 & 84.94 & 49.96 & 13.45 \\
\hline & 15 & 10.42 & 116.57 & 4.69 & 19.41 & 60.22 & 77.11 & 46.49 & 11.21 \\
\hline & 10 & 4.12 & 123.36 & 5.26 & 20.50 & 64.68 & 85.75 & 56.02 & 12.60 \\
\hline & 20 & 9.38 & 130.29 & 4.89 & 29.37 & 88.43 & 85.49 & 74.90 & 12.01 \\
\hline \multirow{4}{*}{ Nubaria } & 16 & 9.88 & 109.34 & 4.59 & 15.19 & 52.57 & 79.46 & 42.06 & 8.29 \\
\hline & 12 & 4.86 & 103.08 & 3.81 & 14.51 & 42.11 & 82.50 & 34.79 & 7.18 \\
\hline & 15 & 7.02 & 103.71 & 3.57 & 13.03 & 41.08 & 71.60 & 29.35 & 7.07 \\
\hline & 17 & 8.04 & 102.39 & 5.13 & 12.74 & 38.45 & 74.92 & 28.89 & 6.13 \\
\hline
\end{tabular}

Table 12: Distributing pattern of 60 genotypes of faba bean into four clusters based on D2 statistics

\begin{tabular}{|c|c|c|c|c|}
\hline \multirow{2}{*}{$\begin{array}{l}\text { No. of } \\
\text { Cluster }\end{array}$} & \multicolumn{2}{|c|}{ NO.G } & \multicolumn{2}{|c|}{ Genotypes included } \\
\hline & Sids & Nub & Sids & Nubaria \\
\hline 1 & 15 & 16 & $\begin{array}{l}\text { G1,G19,G52,G53,G20,G34,G30,G35, } \\
\text { G24,G27,G21,G28,G33,G32,G57. }\end{array}$ & $\begin{array}{l}\text { G1, G2, G6, G42, G23, G59, G18, G19, G41, } \\
\text { G43, G46, G44, G48, G50,G7, G45. }\end{array}$ \\
\hline II & 15 & 12 & $\begin{array}{l}\text { G2,G55,G3,G4,G48,G5,G8,G10,G12, } \\
\text { G15, G18, G26, G23, G44, G58. }\end{array}$ & $\begin{array}{l}\text { G8,G52,G17,G49,G33,G47,G40,G10, } \\
\text { G11,G12, G51, G53. }\end{array}$ \\
\hline III & 10 & 15 & $\begin{array}{l}\text { G9,G17,G56,G11,G31,G25,G59,G39, } \\
\text { G29, G36. }\end{array}$ & $\begin{array}{l}\text { G3,G4,G35,G58,G34,G39,G54,G5,G60,G16, } \\
\text { G38, G56, G26, G55, G57. }\end{array}$ \\
\hline Iv & 20 & 17 & $\begin{array}{l}\text { G6,G46,G41,G43,G37,G54,G7,G38, } \\
\text { G22,G49,G42,G13,G47,G40,G14,G4 } \\
\text { 5,G50,G51, G16, G60. }\end{array}$ & $\begin{array}{l}\mathrm{G} 9, \mathrm{G} 30, \mathrm{G} 13, \mathrm{G} 14, \mathrm{G} 15, \mathrm{G} 20, \mathrm{G} 25, \mathrm{G} 21, \mathrm{G} 28, \\
\mathrm{G} 22, \mathrm{G} 27, \mathrm{G} 31, \mathrm{G} 36, \mathrm{G} 32, \mathrm{G} 37, \mathrm{G} 24, \mathrm{G} 29 .\end{array}$ \\
\hline
\end{tabular}

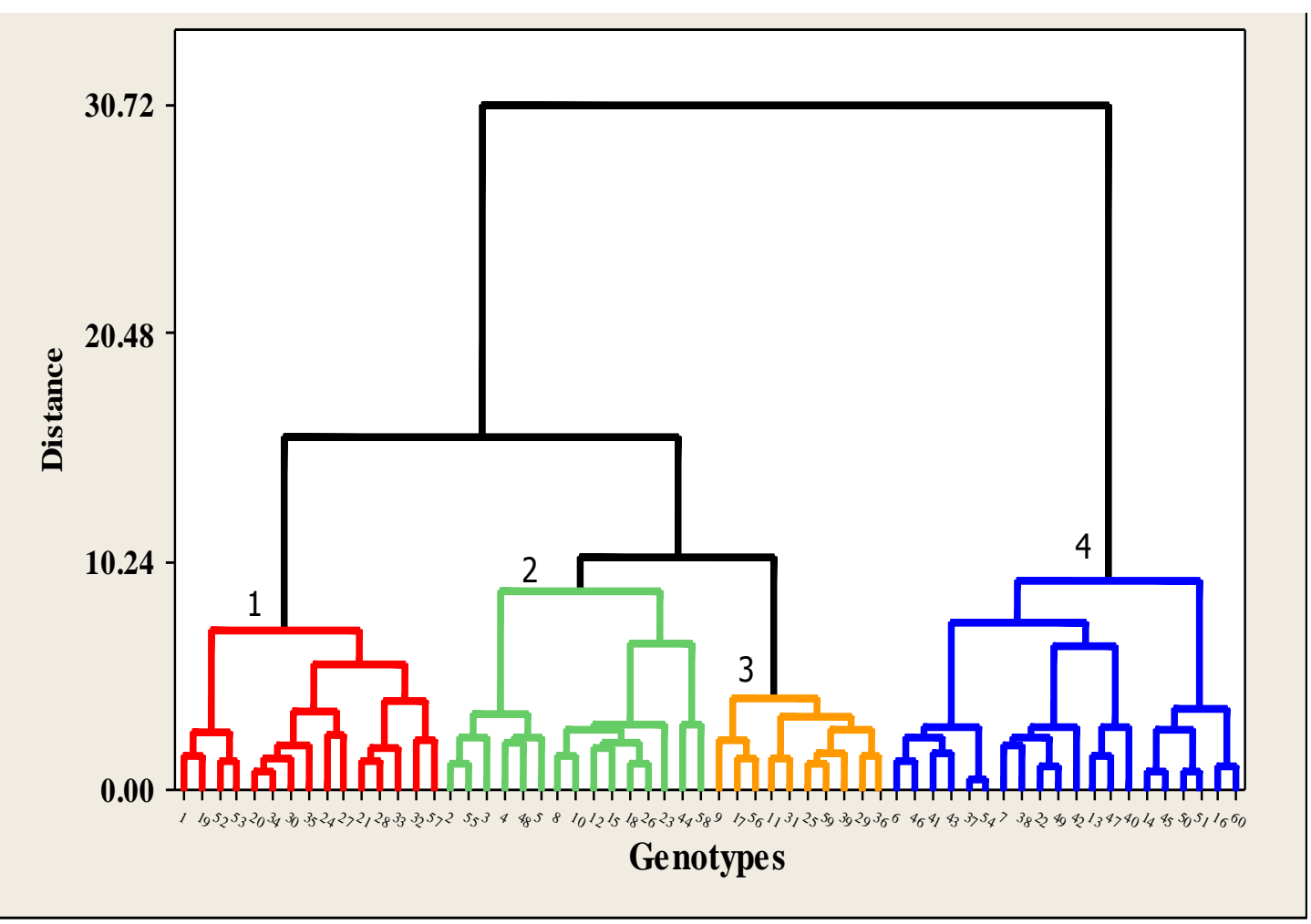

Fig. 1: Dendrogram of 60 faba bean genotypes in sids basd on seven characters 


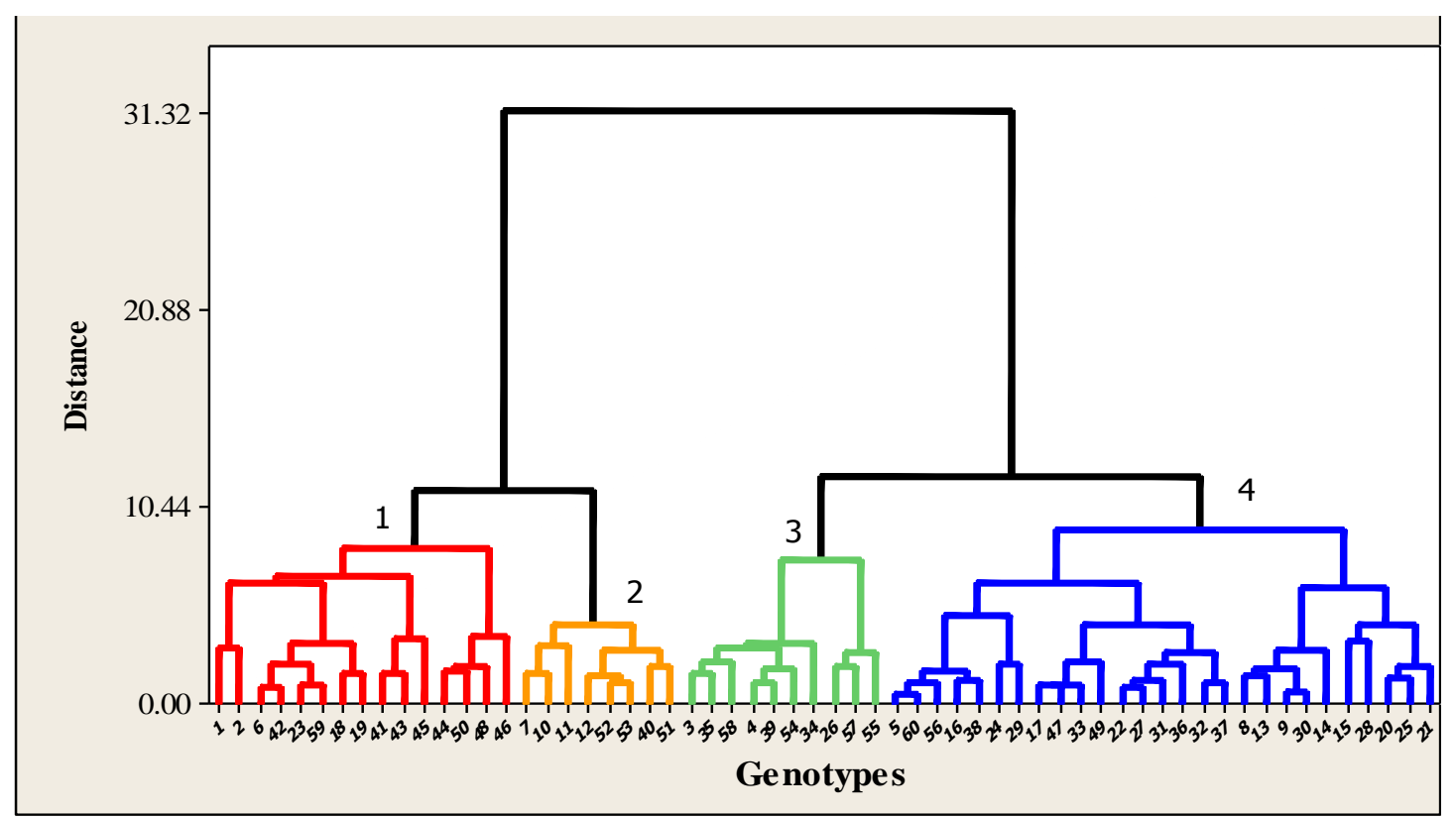

Fig. 2: Dendrogram of 60 faba bean genotypes in Nubaria based on seven characters

\section{CONCLUSIONS}

The studied faba bean genotypes showed variability in most of the studied traits. Overall, the genotypes 27, 52 and 53 at Sids and 45,50 and 55 at Nubaria gave the highest values of seed yield $\left(\operatorname{ardab} . \mathrm{fad}^{-1}\right)$. So this might be useful in identifying promising genotypes of yield potential to breeding programs.

\section{REFERENCES}

Abd El-Mohsen A. A. and S. R. Abo-Hegazy (2013). Comparing the relative efficiency of two experimental designs in wheat field trials. Sci. Res. Rev. J., 1(3): 101-109.

Abd El-Shafi M. A. (2014). Efficiency of classical complete and incomplete block designs in yield trial on bread wheat genotypes. Res. J.Agric and Bio. Sci.,10 (1): 17-23.

Al Ghamdi, S., (2007).Genetic behavior of some selected faba bean cultivars Afr. Crop Sci.Proc., 8:709-714

Al-Rifaee, M. K., (1999). Effect of seed size and plant population density on yield and yield components of local faba bean. M.Sc. Thesis, Jordan Univ. of Sci. and Tech., Irbid, Jordan

Bakry, B.A., T.A. Elewa, M.F. El karamany, M.S. Zeidan and M.M. Tawfik (2011). Effect of row spacing on yield and its components of some faba bean varieties under newly reclaimed sandy soil condition. World Journal of Agricultural Science, 7(1): 68-72.
FAO stat (2012). Food and Agriculture Organization. AvailableWebsite: $<$ http://faostat.fao.org>

Gong YM, Xu SC, Mao WH, Li ZY, HU QZ, ZHANG GW, Ju DING (2011). Genetic diversity analysis of faba bean (Vicia faba L.) based on EST-SSR markers. Agr. Sci. China 10(6):838-844 Khierallah

Hebblethwaite, P.D., J. Ingram, R.K. Scott, and T.J. Elliott, (1977). Some factors influencing yield varieties in field beans (Vicia faba L.). In: Thompson, R. (ed.), Proc., Symp. on the Production, Processing and Utilization of the field bean (Vicia faba L.). pp: 10-16.

Kempton R. A., Seraphin J. C. and Sword A. M.(1994). Statistical analysis of two dimensional variations in variety yield trials. J. Agric. Sci. Cambridge, 122: 335-342.

Lin, C.S.; Binns, M. R.; Voldeg, H. D. and Guillemett, R. (1993). Performance of randomized block designs in field experiments. Agron. J., 85: 168-171.

Masood, M. A.; K. Farooq; Y. Mujahid and M. Z. Anwar (2008). Improvement in precision of agricultural field experiments through design and analysis. Pakistan J.Life Soc. Sci., 6: 8991.

Masood, M. A.; S. N. Malik; N. Nazakat and S. Abid (2007). Blocks within replication improve experimental efficiency in preliminary yield trial on groundnut. Pakistan J. of Agric. Res., 20 (3-4): 116-118. 
Mona, Ismail, Nemat, A. Noureldin , H. S. SaudyManal, M. Mohamed and W. M. Fares (2018). Using Of Alpha Lattice Design for Increasing Precision of Faba Bean Yield Trials. J. Environ. Sci, 44( 2):81-91.

Patterson H. D. and Hunter E. A. (1983).The efficiency of incomplete block designs in national list and recommended list of cereal variety trials. J. Agric. Sci., Camb., 101(2): 427-433.

Patterson H.D. and E.R. Williams (1976). A new class of resolvable incomplete block designs. Biometrika, 63: 83-90.

Payne R. W., D. A. Murray, S. A. Harding, D.B. Baird and D.M. Soutar (2011).An introduction to GenStat for windows (14thEdition).VSN International, Hemel Hempstead, UK.

Qiao, C. G.; Basford, K. E.; Delay, I. H. and Cooper, M. (2000). Evaluation of experimental designs and spatial analysis in wheat breeding trials.Theor. Appl. Genet., 100: 9-16.
Rehab A. M. Abd El-Rahman (2014). Effect of plant population and distribution on yield and yield components of five faba bean genotypes .J. Plant Production, Mans.Univ., 5 (11): 1965 1972.

Silim, S. N., and M. C. Saxena (1992). Comparative performance of some faba bean (Viciafaba L) cultivars of contrasting plant types. 2. Growth and development in relation to yield. Journal of

Vural $\mathrm{H}$ and A. Karasu (2007). A quantitative approach on the evaluation of agronomical characteristics of some cowpea varieties (Vignaun guilata L.) growth in Turkey.World J Agri Sci. 3 (5): 593-596.

Zarea-Fizabady A. and M. Ghodsi (2004). Evaluation of yield and yield components of facultative and winter bread wheat genotypes (Triticum aestivum L.) under different irrigation regimes in Khorasam Province in Iran. J. Agron., 3(3): 184-187. 


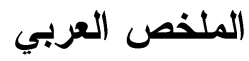

\section{تقييم التباين بين تر اكيب الوراثية من الفول البلاي في بيئات مختلفة \\ رحاب أحمد محمد عبد الرحمن'، أبوزيد عبد المحسن أبوزيد'، وفاء وهبه محمد الثافعيץ \\ 'قسم بحوث الححاصيل البقولية - معهد بحوث المحاصيل الحقلية - مركز البحوث الزراعية.

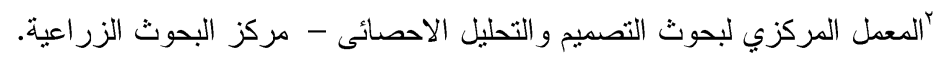

يعتبر تطوير انتاج اصناف عالية المحصول الهدف الاساسي فى معظم برامج التزبيه لمحصول الفول البلــدي، وتهدف هذه الدراسة الى تقييم ستون تركيب وراثي من الفول البلدى لمحصول البذور ومكوناته تحت ظروف بيئية مختلفة بكل من محطة البحوث الزراعية بسدس (منطقة مصر الوسطي) ومحطة بحوث النوبارية منطقة شمال الوجه

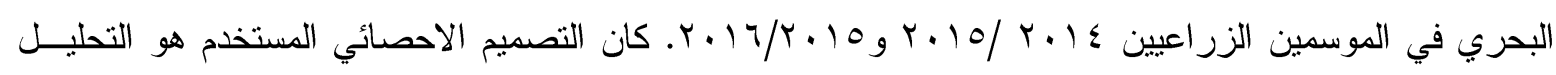

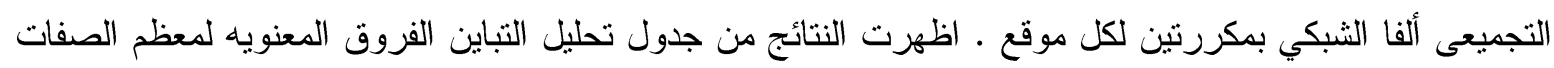

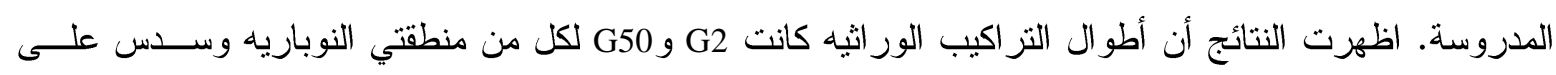
الترتيب، كما اوضحت النتائج التي تم الحصول عليها أن التركيب الوراثي G42 قد أعطى أعلى القيم بالنسبة لعـدند

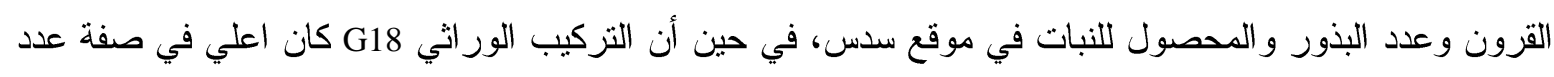

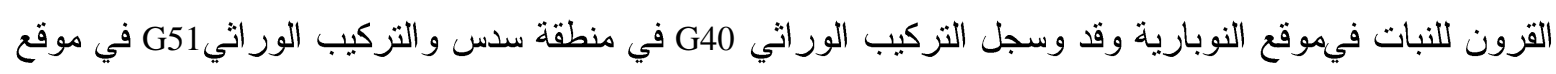

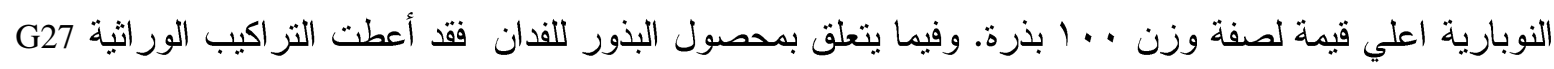

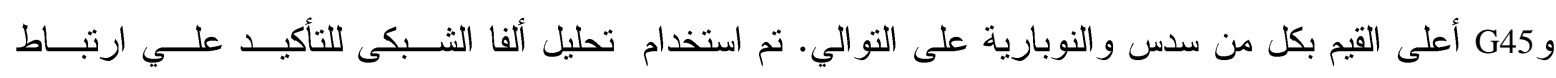
مجموعات من التر اكيب الور اثية معا فى الصفات تحت الدراسة بشكل مباشر أو غير مباشر ـ كما أتضح من نتــائج

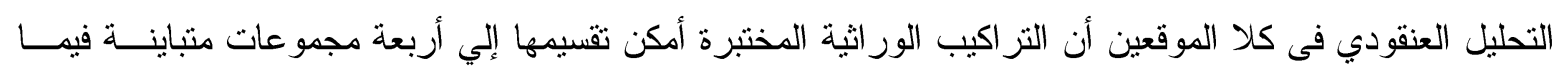

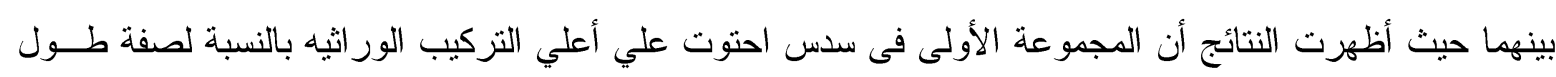

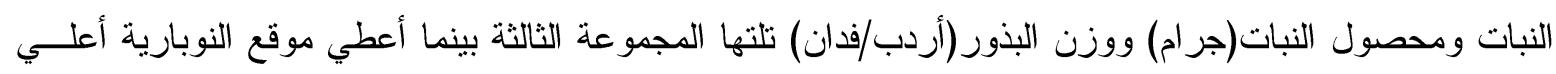

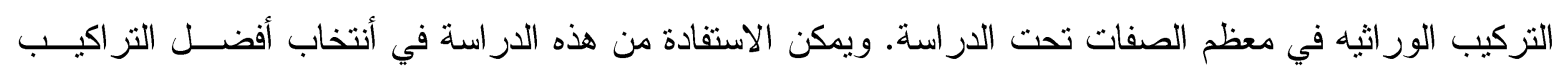

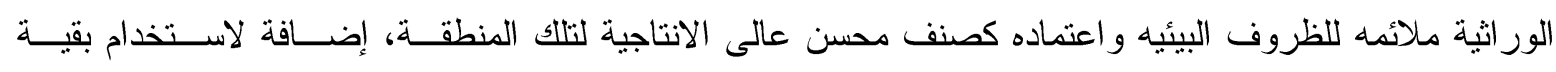

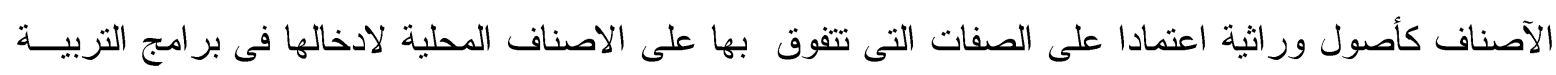

